\title{
Die Haftung von Partnern einer Partnerschaftsgesellschaft für berufliche Fehler
}

VERONIKA JÅGER

Die Haftung von Partnern einer Partnerschaftsgesellschaft

für berufliche Fehler

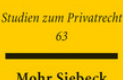

Mohr Siebeck

2017. XXII, 283 Seiten. StudPriv 63

ISBN 978-3-16-155168-0

DOI 10.1628/978-3-16-155168-0

eBook PDF $89,00 €$

ISBN 978-3-16-155167-3

fadengeheftete Broschur 89,00€
Bei der Wahl der geeigneten Rechtsform zur Berufsausübung kommt der persönlichen Haftung und der Haftungsbeschränkung eine entscheidende Bedeutung zu. Für Angehörige Freier Berufe eröffnet die

Partnerschaftsgesellschaft die Möglichkeit, die Haftung für Schäden infolge fehlerhafter Berufsausübung auf einzelne Partner zu beschränken bzw. sie durch die Wahl der Partnerschaftsgesellschaft mit beschränkter Berufshaftung vollständig auszuschließen.

Wann wird die Schwelle haftungsrelevanten Verhaltens überschritten? Auf wen kann sich die persönliche Haftung konzentrieren? Und welche (ungeschriebenen) Voraussetzungen müssen erfüllt sein, damit die persönliche Haftung für berufliche Fehler bei einer Partnerschaftsgesellschaft mit beschränkter Berufshaftung wirksam ausgeschlossen ist? Veronika Jäger untersucht diese Fragen eingehend und entwickelt Abgrenzungskriterien und Definitionen, mit deren Hilfe das Ausmaß der persönlichen Haftung kalkulierbarer wird.

Veronika Jäger Geboren 1989; Studium der Rechtswissenschaften an der Universität Bayreuth mit Wirtschaftswissenschaftlicher Zusatzausbildung; wissenschaftliche Hilfskraft an der Universität Bayreuth; 2016 Promotion; seit 2016 Referendarin am Landgericht München II.
Jetzt bestellen:

https://mohrsiebeck.com/buch/die-haftung-von-partnern-einer-partnerschaftsgesellschaft-fuer-berufliche-fehler-

9783161551680?no_cache=1

order@mohrsiebeck.com

Telefon: +49 (0)7071-923-17

Telefax: $+49(0) 7071-51104$ 